\title{
La participación social de los jóvenes españoles
}

\author{
OSCAR A. SANTACREU FERNÁNDEZ \\ INSTITUTO UNIVERSITARIO DE DESARROLLO SOCIAL Y PAZ \\ UNIVERSIDAD DE ALICANTE
}

\section{Resumen}

Este trabajo analiza la participación social de los jóvenes españoles, partiendo del concepto de capital social. Utilizando como metodología los modelos estructurales de covarianzas, busca la relación entre las actividades de participación de los jóvenes y factores como la pertenencia a asociaciones y colectivos, el interés que manifiestan por la política, el grado de confianza en la utilidad de la participación política y valores como la solidaridad. También evalúa la influencia de variables externas como el género, la clase social o los estudios. La principal hipótesis que se plantea consiste en que el grado de participación general está mediada principalmente por el interés en la política y por la actividad en ámbitos de participación institucionalizados.

Palabras clave: juventud, participación, capital social, política, modelos estructurales de covarianzas.

\begin{abstract}
This paper analyzes the social participation of Spanish young people, based on the concept of social capital. We use Covariance Structural Models as methodology to find the relationship between the participation activities of young people and factors such as their associations membership, their interest on politics, their confidence feeling on the political participation and values such as solidarity. It also evaluates the influence of external variables such as gender, social class or studies. The main hypothesis is that the overall participation is mediated primarily by the interest in politics and by the activity in institutionalized areas of participation.
\end{abstract}

Keywords Youth, participation, social capital, politics, Covariance Structural Models 


\section{La participación social}

La participación social es, sin duda, uno de los temas más importantes en los estudios sociales. De su importancia se ha llegado a afirmar que conforma un "sector desconocido" (Nielsen, 1979). Por ejemplo, en la Unión Europea se estima que cerca de cien millones de personas pertenecen al menos a una organización no gubernamental

(Comisión de las Comunidades Europeas, 1997; Jarré, 1991). Concretamente en España, durante la década de los 90 se ha producido un crecimiento espectacular de la participación, pasando del 25,2\% que mostraba el Eurobarómetro de 1987 que nos situaba como el último país en participación después de Italia- (Eurostat, 1991), a un grado de asociacionismo entorno al 40\% (Alberich, 1994; Ruiz, 2000).

El grado de asociacionismo juvenil en España no difiere demasiado del grado de asociacionismo de la población general (Martín, 1991). La mayor parte del asociacionismo se concentra en asociaciones voluntarias (29\%), mientras que sólo un $2 \%$ de los jóvenes están afiliados a algún partido político, y un 3\% manifiesta estar afiliado a algún sindicato (Prieto, 1993). Algunos autores destacan que existe un gran potencial para el voluntariado entre los jóvenes, dado que más del $50 \%$ se ha planteado esta forma de participación (Orizo, 1995)

Evidentemente, al hablar de participación no debemos limitarnos únicamente a los espacios convencionales, formales o institucionalizados de participación. Hay diversos estudios (Crozier et al., 1975; Barnes and Kaase, 1979) sobre las formas de participación no convencional, originariamente asociadas con formas de protesta como boicots a determinados productos, por ejemplo, y que en la actualidad se han convertido en formas comunes de participación individual (Dalton 1999, 2004; Norris, 1999). Se trata de formas de participación emergentes a partir de un cambio en los valores cívicos (Inglehart, 1990). Como se verá, una de nuestras hipótesis será el nexo explicativo que existe entre la participación en asociaciones y el nivel de participación general que abarca otras formas no convencionales de participación. De hecho determinados estudios sugieren una relación de reciprocidad y refuerzo entre formas de participación convencional y no convencional (Ganuza y Francés, 2008)

Este trabajo presenta la relación entre los valores de los jóvenes españoles y su participación social, entendida como interacción de actores sociales, individuales en este caso, en situaciones y procesos más o menos estructurados con unos fines significativos y con unas motivaciones que pueden ir desde la satisfacción de sus intereses (instrumentales) hasta el reconocimiento de su subjetividad, pasando por la acción pública promotora.

Analizar el fenómeno de la participación social únicamente a partir del grado de asociacionismo nos ofrece, como hemos comentado, una visión parcial del fenómeno. En este sentido, un concepto importante para el análisis de la participación social en particular, y para el diagnóstico de fenómenos sociales en general, es el de capital social. Existen numerosas definiciones, como la del Banco 
Mundial que entiende el capital social como "las instituciones, relaciones y normas que conforman la calidad y cantidad de las interacciones sociales de una sociedad. Numerosos estudios demuestran que la cohesión social es un factor crítico para que las sociedades prosperen económicamente y para que el desarrollo sea sostenible. El capital social no es sólo la suma de las instituciones que configuran una sociedad, sino que es asimismo la materia que las mantiene juntas" (Banco Mundial, 1999). También ha sido definido como el entramado de interacciones estables en las que se incardina un individuo o actor social (Putnam, 2000). Como diría Hilarie Owen, no sólo es importante lo que sabes, sino a quién conoces. Las aportaciones clásicas a la definicón de capital social de Bourdieu (1984), Coleman (1988), Putnam (2000) y Fukuyama (2000) han sido enriquecidas posteriormente por los trabajos de Lechner (2000), Rits (2000), Durston (2000/2001), Flores y Rello (2001), entre otros.

En este trabajo la definición de capital social recoge las normas y valores compartidos, el reconocimiento y la aceptación mutua, los valores sociales (en particular la confianza), el compromiso cívico expresado en la reciprocidad, la participación social y la construcción de redes sociales. A pesar del amplio campo de reflexión que esta definición plantea, en este trabajo vamos a centrarnos en una serie de cuestiones básicas como las siguientes:

- ¿Las actividades de participación de los jóvenes vienen explicadas por la pertenencia a asociaciones y colectivos?

- ¿Hay relación entre género, clase social, estudios... y participación?

- ¿Qué papel juegan en la participación valores como la solidaridad?

- ¿Hay relación entre el interés por la política y la participación social?

Para tratar de responder a estas preguntas utilizaremos los datos del estudio sobre Ciudadanía y Participación, integrado en el International Social Survey Programme (ISSP). Tomaremos como ámbito España y, por tanto, la encuesta realizada por el CIS del 8 al 18 de octubre de 2004.

\section{Análisis comparativo por edad}

Existe disparidad de criterios a la hora de fijar la edad a la que un individuo deja de pertenecer al colectivo de la juventud. En general hay cierto nivel de consenso en que la juventud es "una etapa de transición entre la infancia y la edad adulta. Los límites de edad no son precisos, habitualmente se considera que se inicia con la pubertad y concluye con la independencia de la tutela de la familia y la asunción de roles y status propios de la madurez, muchos de ellos relacionados con el ámbito de la producción" (Uña, O.; Hernández, A.; Prado, J.M., 2004:1399). Por motivos operativos en este trabajo es necesario fijar una edad máxima, de modo que usaremos el mismo criterio que la normativa europea de programas juveniles, esto es, fijamos la frontera en los 35 años. Así, este análisis busca diferencias respecto a la participación comparando por un lado el grupo de individuos de 35 años y menos (los jóvenes) y por otro el grupo de los mayores de 35 años. 
La primera conclusión a la que se puede llegar es la existencia de unos valores compartidos. La siguiente tabla nos muestra lo que podría ser un "manual de buen ciudadano": votar siempre en las elecciones, no intentar evadir impuestos, obedecer las leyes y normas... Vemos que no existen diferencias significativas entre los dos grupos de edad salvo en el campo de la participación y en la disponibilidad para servir en el Ejército en tiempo de necesidad, dos aspectos en los que los menores de 36 años presentan unas medias ligeramente mayores.

Tabla 1. Formas de participación

\begin{tabular}{|c|c|c|c|c|}
\hline & \multicolumn{4}{|c|}{ Edad (Categorizada) } \\
\hline & \multicolumn{2}{|c|}{$<=35$} & \multicolumn{2}{|c|}{$36+$} \\
\hline & Media & Desv. típica & Media & Desv. típica \\
\hline Votar siempre en las elecciones & 5 & 2 & 6 & 1 \\
\hline No intentar evadir impuestos nunca & 6 & 1 & 6 & 1 \\
\hline Obedecer siempre las leyes y normas & 6 & 1 & 6 & 1 \\
\hline $\begin{array}{l}\text { Mantenerse informado sobre las } \\
\text { acciones del Gobierno }\end{array}$ & 5 & 2 & 5 & 2 \\
\hline $\begin{array}{l}\text { Participar en asociaciones de carácter } \\
\text { social o político }\end{array}$ & 4 & 2 & 5 & 2 \\
\hline $\begin{array}{l}\text { Tratar de entender las ideas de la gente } \\
\text { con opiniones distintas a las nuestras }\end{array}$ & 6 & 1 & 6 & 1 \\
\hline $\begin{array}{l}\text { Elegir artículos de consumo que, } \\
\text { aunque sean algo más caros, no dañen } \\
\text { el mediombiente }\end{array}$ & 5 & 2 & 5 & 2 \\
\hline $\begin{array}{l}\text { Ayudar a gente que, en nuestro país, } \\
\text { vive peor que } \mathrm{Ud} \text {. }\end{array}$ & 6 & 1 & 6 & 1 \\
\hline $\begin{array}{l}\text { Ayudar a gente que, en otras partes del } \\
\text { mundo, vive peor que Ud. }\end{array}$ & 6 & 1 & 6 & 1 \\
\hline $\begin{array}{l}\text { Estar dispuesto a servir en el Ejército } \\
\text { en tiempo de necesidad }\end{array}$ & 3 & 2 & 4 & 2 \\
\hline
\end{tabular}

En todo caso nuestro interés se centrará a continuación en tratar de determinar qué aspectos pueden intervenir a la hora de explicar el grado de participación de los jóvenes. Así, trataremos de comprender la influencia en este sentido de la actividad en espacios institucionalizados de participación, del interés por la política y de la autopercepción de la utilidad de la participación. También buscaremos la posible influencia de valores asociados a la juventud como la confianza o la solidaridad (INJUVE, 1996; Soler, 2007). Por último buscaremos la influencia de variables como el género, la clase social o el nivel de estudios. La multitud de factores intervinientes en la participación aconseja un análisis multivariante, que presentamos a continuación.

\section{Modelo de participación}

Como hemos comentado, a partir del breve análisis descriptivo expuesto tenemos la posibilidad de realizar un análisis multivariante sobre la participación de los jóvenes. En este sentido se puede aplicar un modelo estructural de covarianzas. Esta técnica estadística multivariante se suele utilizar para estudiar y analizar las relaciones de dependencia, en términos de carga explicativa, entre las variables que 
consideramos que forman parte de un fenómeno social determinado, la participación social en este caso. El método define un modelo como conjunto de ecuaciones estructurales que quedan representadas en un diagrama causal.

El primer paso será, por tanto, seleccionar las variables que formarán parte de ese sistema. Este proceso de selección tiene como limitación, evidentemente, la información disponible en la base de datos que vamos a utilizar (estudio sobre Ciudadanía y Participación del CIS, 2004). Por otro lado, escogeremos las variables en función de su correspondencia con algunas de las dimensiones previamente citadas al hablar de capital social y participación social. En ese sentido, las variables que se consideran en el modelo son las siguientes:

- Pertenencia activa a alguna asociación durante el último año.

- Grado de participación activa durante el último año.

- Grado de interés por la política.

- Escepticismo político, entendido como la falta de confianza en las posibilidades reales del individuo para influir en la política.

- Confianza en el gobierno.

- Confianza general en la sociedad.

- Solidaridad (disposición para ayudar a las personas que lo necesiten).

A estas variables hemos de añadir, a efectos de control, las variables género, clase social y estudios. Tal y como se explica más adelante, dado que centraremos el análisis en la población menor de 36 años no utilizaremos la variable edad como variable explicativa.

Nos planteamos, por tanto, que la pertenencia a asociaciones, el interés por la política, los valores sociales, la participación política, la solidaridad o la clase social tienen un efecto positivo a la hora de explicar la participación social. En particular, nuestra principal hipótesis consiste en que el grado de participación general está mediada principalmente por el interés en la política y por la actividad en ámbitos de participación institucionalizados. El nivel de estudios y la disposición a ayudar, a dedicar tiempo a los demás, influirán también directamente en el grado general de participación. Al hablar del nivel de estudios, cabe comentar su relación también directa con el interés por la política desde un punto de vista activo que considera la posibilidad de que este interés sirve realmente para algo. Paralelamente, se espera una relación positiva también entre el nivel de estudios y la pertenencia a alguna asociación. En cuanto a la clase social, es de esperar que las clases más bajas sean más escépticas respecto a su capacidad para influir en política.

Estas hipótesis relacionales se plasman en el modelo identificado, consistente y recursivo que representa el siguiente gráfico.

Los valores en las flechas corresponden a los valores de las pruebas $t$, e indican que las relaciones establecidas entre las variables son estadísticamente significativas. Los coeficientes de bondad del ajuste nos muestran también que las diferentes hipótesis dan lugar a una explicación consistente. Así, el chi-cuadrado, una medida de ajuste global del modelo, es ajustado comparado a los grados de 
libertad, indicando un buen ajuste. Otros indicadores que complementan la dependencia de chi-cuadrado del tamaño muestral expresan también un ajuste razonable de nuestra explicación a partir de los datos disponibles ${ }^{1}$.

Gráfico 1. Modelo estructural sobre la participación.

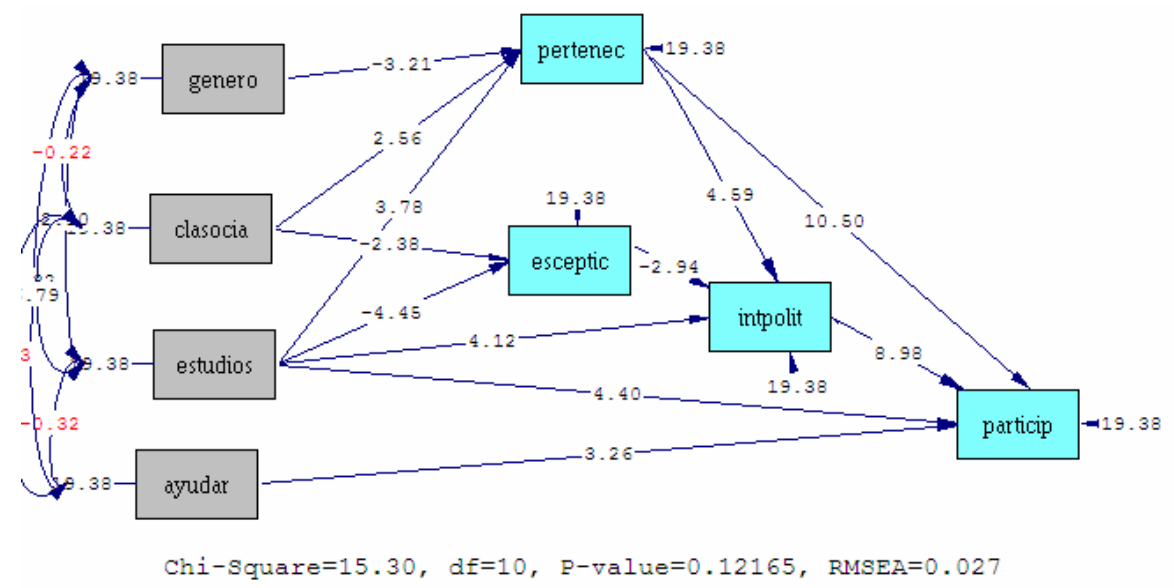

Como

variables exógenas consideramos el género, la clase social, el nivel de estudios y la disposición para ayudar (solidaridad). Por otro lado, como se ha dicho, el hecho de incluir en el modelo únicamente la población menor de 36 años hace que los datos no nos permitan establecer una relación estadísticamente significativa de la variable género con el resto de variables estudiadas, por lo que dicha variable no ha sido considerada para ser incluida en el modelo.

Se observa también que la variable central en el modelo es la que se refiere al interés por la política, en tanto que tiene un importante papel como mediadora para explicar la relación de las otras variables con la variable que recoge el grado de participación. También influyen directamente la pertenencia efectiva a asociaciones y la solidaridad como compromiso cívico.

La utilización de un modelo estructural de covarianzas nos permite determinar en qué grado los datos sustentan la hipótesis de partida. A continuación mostramos las ecuaciones de dicho modelo.

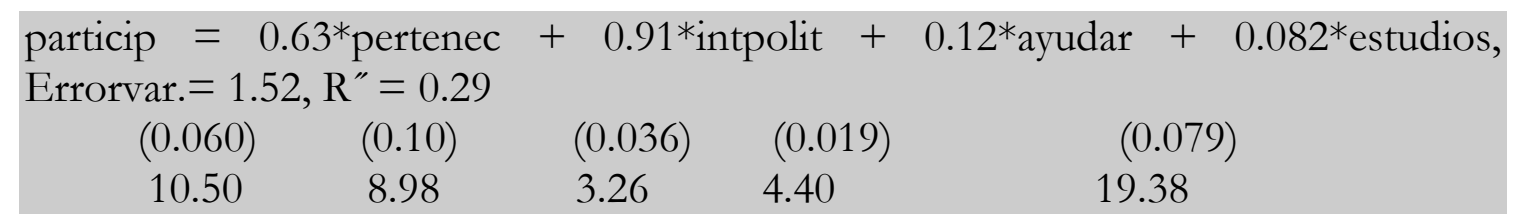

\footnotetext{
${ }^{1}$ P .12165 y RMSEA=0.027. Los índices GFI (Goodness of Fit Index) y AGFI (Adjusted Goodness of Fit Index) presentan unos valores de 0.99 y 0.98 respectivamente.
} 
Tal y como habíamos planteado, la participación vendría explicada de forma directa por la pertenencia a asociaciones, el interés por la política, la disposición a ayudar y el nivel de estudios, siendo el interés por la política la variable que mayor carga explicativa muestra (0.91). Apreciamos también cómo las pruebas $t$ para cada coeficiente nos indican que las relaciones propuestas son significativas $(10.50,8.98$, 3.26 y 4.40 respectivamente).

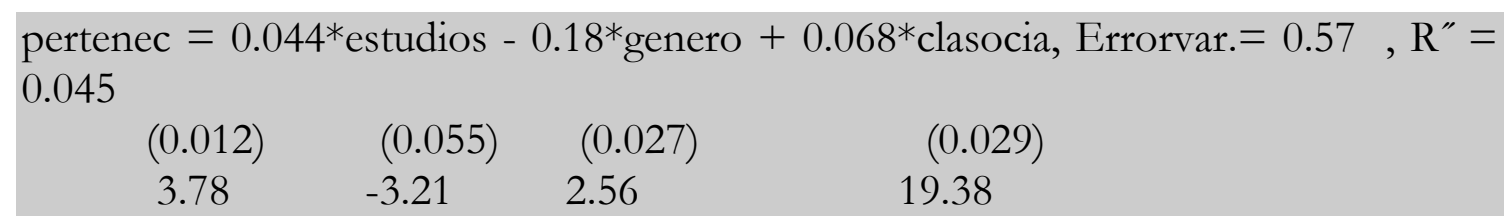

Por su parte, la pertenencia a asociaciones de los jóvenes españoles viene explicada por el género, la clase social y el nivel de estudios, en todos los casos de forma positiva, lo que nos daría un perfil de un hombre de clase social media-alta y nivel de estudios medio-alto, siempre hablando en términos probabilísticos.

intpolit $=0.097 *$ pertenec $-0.097 *$ esceptic $+0.028 *$ estudios, Errorvar. $=0.20, \mathrm{R}^{\prime \prime}=$
0.074 C
$\begin{array}{cccc}(0.021) & (0.033) & (0.0067) & (0.010) \\ 4.59 & -2.94 & 4.12 & 19.38\end{array}$

Por otro lado, el interés por la política resulta especialmente importante en este análisis dado que se trata de una variable mediadora a través de la cual influyen en la participación social otras variables. En este modelo, el interés por la política será probablemente mayor si se pertenece a alguna asociación, se tiene un nivel de estudios medio-alto y un bajo escepticismo en la política. También recibe el efecto de la variable género a través de la pertenencia o no a asociaciones, como hemos visto, así como de la clase social y del nivel de estudios a través del escepticismo: el escepticismo es mayor en clases sociales bajas y un mayor escepticismo explica un menor interés por la política.

De este modo el escepticismo, que se entiende aquí como la falta de confianza en las posibilidades reales del individuo para influir en la política, viene explicado en el modelo principalmente por la clase social (mayor escepticismo en las clases sociales bajas) y por un menor nivel de estudios.

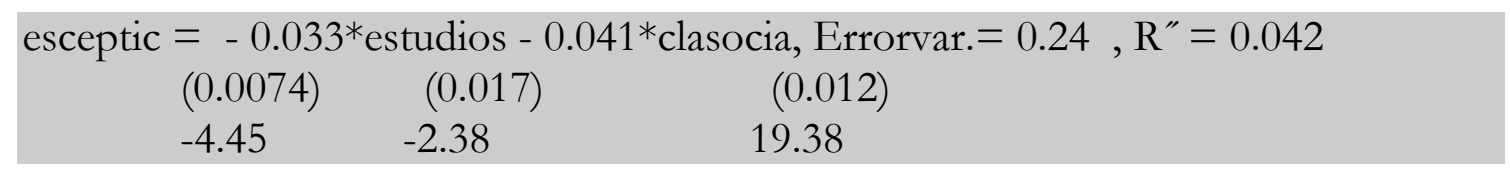

En cuanto al género, observamos que presenta un doble efecto sobre la participación. La principal variable mediadora es la pertenencia a asociaciones, que puede ir unida o no a un mayor interés por la política. Por otro lado es cierto que, en términos absolutos, las mujeres participan más que los hombres $(58,3 \%$ frente al $54,3 \%$ ), pero los hombres presentan más participaciones múltiples (entendidas como la participación durante el último año en más de cinco actos), lo que se explica 
por la mayor pertenencia activa de los hombres a asociaciones $(38,4 \%$ frente al $23,5 \%$ de las mujeres).

La clase social media-alta también tiene un efecto indirecto positivo sobre la participación a través de la mayor pertenencia a asociaciones y de una mayor confianza en la efectividad de las acciones políticas y el consiguiente mayor interés por la política.

Por último, el mayor nivel de estudios es sin duda la variable que más efectos tiene sobre la participación: al efecto directo hay que sumar un efecto indirecto a través de un mayor interés por la política, a través de un menor escepticismo y el consiguiente mayor interés por la política, y otro efecto indirecto mediante la mayor pertenencia a asociaciones.

\section{Bibliografía}

ALBERICH, T. (1994) "Aspectos cuantitativos del asociacionismo en España", en Mundo asociativo. Documentación Social No 94 . Madrid.

BANCO MUNDIAL (1999). Capital social. En: http://go.worldbank.org/789EMK6WV0

BARNES, S. H. AND KAASE, M. (Eds) (1979). Political Action. Mass Participation in Five Western Countries. Beverly Hills, CA: Sage.

BOURDIEU, P. (1984) Questións de Sociologie. Paris: Les Éditions de Minut

COLEMAN, J. (1988) "Social Capital in the Creation of Human Capital". American Journal of sociology. University of Chicago, Vol. 94.Supplement S95-S120.

COMISIÓN DE LAS COMUNIDADES EUROPEAS (1997) El fomento del papel de las asociaciones y fundaciones en Europa. Comunicación de la Comisión, Bruselas, 06.06.1997. $\mathrm{COM}(97) 241$ final.

CROZIER, M., HUNTINGTON, S. P. and Watanuki, J. (1975). The Crisis of Democracy. New York: New York University Press.

DALTON, R. J. (1999). "Political support in advanced industrial democracies." In Norris, P. (Ed.), Critical Citizens: Global Support for Democratic Governance. Oxford: Oxford University Press, pp. 57-78.

DURSTON, J. (2000) ¿Que es el Capital Social Comunitario? CEPAL. Serie Políticas Sociales, 33. Santiago de Chile.

EUROSTAT (1991) Retrato Social de Europa. Oficina Estadística de las Comunidades Europeas - Eurostat.

FLORES, M. e RELLO, F. (2001) “Capital Social: virtudes e limitações” CEPAL. Conferencia En busca de un nuevo paradigma: capital social y reducción de la pobreza en América Latina y el Caribe. Santiago de Chile, 24 a 26 de sep. 2001. 
FUKUYAMA, F. A (2000) Grande Ruptura. Trad. Nivaldo Mantingelli Jr. Rio de Janeiro: Rocco.

GANUZA, E Y FRANCÉS, F. (2008) "Rethinking the sources of participation: a case study of Spain" en European Sociological Review, Vol. 24, no 4, september 2008. Pags 479-493. Oxford University Press.

INGLEHART, R. (1990). Cultural Shift in Advanced Industrial Society. Princeton: Princeton University Press.

INGLEHART, R. (1997). Modernization and Postmodernization: Cultural, Economic and Political Change in 43 Societies. Princeton: Princenton University Press.

INJUVE (1996) La solidaridad de la juventud. Madrid: Instituto de la Juventud.

JARRÉ, D. (1991) "La iniciativa social y humanitaria en Europa Occidental", en Las entidades no lucrativas de carácter social y bumanitario. Colección solidaridad. $\mathrm{N}^{\mathrm{0}} 1$, Fundación ONCE, Madrid

LECHNER, N. (2000) "Desafíos de un desarrollo humano: individualización y capital social” En KLIKSBERG, B. e TOMASSINI, L. Capital Social y Cultura: Claves estratégicas para el desarrollo. Argentina: BID y Fondo de Cultura Económica de Argentina, S.A., p.19-58.

MARTÍN, M. (1991) Los valores actuales de la juventud en España. Ministerio de Asuntos Sociales, Instituto de la Juventud. Madrid.

NIELSEN, W (1979) The endangered sector. Columbia University Press, New York

NORRIS, P. (1999). "Conclusions: The growth of critical citizens and its consequences." In Norris, P. (Ed.) Critical Citizens: Global Support for Democratic Governance. Oxford: Oxford University Press.

ORIZO, F.A. (1995) Dinámica intergeneracional en los sistemas de valores de los Españoles. Centro de Investigaciones Sociológicas, Madrid.

PRIETO, R. (1993) "Asociacionismo, ideología y participación". En Navarro López y Mateo Rivas, Informe Juventud en España 92. INJUVE, Madrid.

PUTNAM, R. D. (2000) Comunidade e Democracia: a experiência da Itália Moderna. Trad. Luiz Alberto Monjardim, 2.Ed. Rio de Janeiro: Editora FGV.

RITS, G. (2000) "La cultura y el capital social: cómplices o víctimas del desarrollo?" En KLIKSBERG, B. y TOMASSINI, L. Capital Social y Cultura: Claves estratégicas para el desarrollo. Argentina: BID y Fondo de Cultura Económica de Argentina, S.A., p.129-150.

RUIZ, J.I. (dir) (2000) El sector no lucrativo en España Fundación BBV, Madrid. 
SOLER, P. (2007) Factores psicosociales explicativos del voluntariado universitario. Tesis doctoral. Alicante: RUA. Disponible en:

http:/ /hdl.handle.net/10045/7756

UÑA, O.; HERNÁNDEZ, A.; PRADO, J.M. (2004) Diccionario de Sociología. ESIC Editorial, Barcelona. 